\title{
Determinación del riesgo cardiovascular en una población
}

\author{
Juan Camilo Álvarez-Ceballos ${ }^{a}$, Andrés M. Álvarez-Múñoz ${ }^{\mathrm{a}}$, \\ Wilder Carvajal-Gutiérrez ${ }^{a}$, María Mercedes González ${ }^{a}$, Jorge Luis Duque ${ }^{b}$ \\ y Olga Alicia Nieto-Cárdenas ${ }^{a, *}$
}

\author{
a Grupo de Salud Pública, Universidad del Quindío, Quindío, Colombia \\ b Programa de Medicina, Facultad de Ciencias de la Salud, Universidad del Quindío, Quindío, Colombia
}

Recibido el 13 de junio de 2016; aceptado el 18 de agosto de 2016

Disponible en Internet el 3 de octubre de 2016

\section{PALABRAS CLAVE \\ Enfermedad \\ cardiovascular; \\ Factores de riesgo \\ cardiovascular; \\ Estudios de validación}

\begin{abstract}
Resumen
Objetivo: Determinar el riesgo cardiovascular con las escalas Framingham, Framingham Colombia, Procam y Procam Colombia en la población del programa de riesgo cardiovascular de una institución de salud de primer nivel en la ciudad de Armenia, Quindío.

Métodos: Se realizó un estudio descriptivo de corte transversal, que tomó como población las personas del programa de riesgo cardiovascular de una institución de salud de primer nivel en la ciudad de Armenia, Quindío, entre el 1 de enero y 31 de diciembre de 2015. Se describieron las variables en promedio, desviación estándar e intervalos de confianza, con un análisis comparativo por sexo para los factores de riesgo en cada escala.

Resultados: En la población del programa de riesgo cardiovascular se encontró, un promedio de edad de 64,54 años e índice de masa corporal de $27,71 \mathrm{~m}^{2} / \mathrm{kg}$. El 76,48\% presenta hipertensión arterial, el 20,86\% presenta diabetes mellitus y el 9,36\% fuma.

El riesgo cardiovascular calculado con la escala de Framingham es el 11,36\% y con Framingham Colombia es el $8,52 \%$. Con la escala Procam es el 9,44\% y con Procam Colombia es el $8,81 \%$.

El índice de masa corporal, la edad y el colesterol LDL, no son estadísticamente significativos para la valoración del riesgo con las escalas Framingham y Framingham Colombia.

Conclusiones: Se propone que la escala adecuada para la medición del riesgo cardiovascular en esta población es la escala de Procam o Procam Colombia, porque identifica casi todas las variables de manera significativa. Las escalas de Framingham y Framingham Colombia deben ser utilizadas con precaución en esta población.

(C) 2016 Sociedad Colombiana de Cardiología y Cirugía Cardiovascular. Publicado por Elsevier España, S.L.U. Este es un artículo Open Access bajo la licencia CC BY-NC-ND (http:// creativecommons.org/licenses/by-nc-nd/4.0/).
\end{abstract}

\footnotetext{
* Autor para correspondencia.

Correos electrónicos: olgalicianieto@gmail.com, oanieto@uniquindio.edu.co (O.A. Nieto-Cárdenas).
} 


\section{KEYWORDS}

Cardiovascular

disease;

Cardiovascular risk

factors;

Validation studies

\section{Determining cardiovascular risk in a population group}

\begin{abstract}
Motivation: To determine cardiovascular risk with Framingham, Framingham Colombia, Procam, and Procam Colombia in the population of the cardiovascular risk program of a first level institution in the city of Armenia, Quindío.

Methods: A cross-sectional, descriptive study was conducted that selected as population the participants of the cardiovascular risk program of a first level health institution in the city of Armenia, Quindío, between $1^{\text {st }}$ of January and $31^{\text {st }}$ of December 2015. Average variables, standard deviation and confidence intervals were described with a comparative gender-sensitive analysis of risk factors for each scale.

Results: The average age in the cardiovascular risk program was 64.54 years, and a body mass index of $27.71 \mathrm{~m}^{2} / \mathrm{kg}$. $76.48 \%$ presents arterial hypertension, $20.86 \%$ diabetes mellitus and $9.36 \%$ smoke.

Cardiovascular risk according to the Framingham scale is $11.36 \%$, and with Framingham Colombia it is $8.52 \%$. According to Procam scale it is $9.44 \%$, and with Procam Colombia it is 8.81\%.

Body mass index, age and LDL cholesterol are not statistically significant for risk assessment with Framingham and Framingham Colombia scales.

Conclusion: The proposed appropriate scale for measuring cardiovascular risk in this population group is the Procam or Procam Colombia scale, because it identifies almost all variables in a significant way. Framingham and Framingham Colombia scales must be used with caution for this population group

(c) 2016 Sociedad Colombiana de Cardiología y Cirugía Cardiovascular. Published by Elsevier España, S.L.U. This is an open access article under the CC BY-NC-ND license (http:// creativecommons.org/licenses/by-nc-nd/4.0/).
\end{abstract}

\section{Introducción}

La Organización Panamericana de la Salud plantea que "las enfermedades cardiovasculares son la principal causa de discapacidad y de muerte prematura en todo el mundo, y contribuyen sustancialmente al aumento de los costos de la atención de salud. La lesión fundamental es la aterosclerosis, que se presenta con el transcurso de los años y suele estar avanzada cuando aparecen los síntomas, generalmente, en la madurez"'

Se han identificado factores de riesgo los cuales pueden ser principales o secundarios, son factores de riesgo principales: la hipertensión arterial, la dislipidemia, la diabetes mellitus, la obesidad y el sobrepeso, el tabaquismo, el sedentarismo, el género, la herencia y la edad. Y son factores de riesgo secundarios: el estrés, las hormonas sexuales, los anticonceptivos orales y el consumo de alcohol ${ }^{2}$.

Las enfermedades cardiovasculares son la principal causa de muerte en el mundo ${ }^{3}$. Se calcula que en el año 2012 murieron por esta causa 17,5 millones de personas, lo cual representa un $31 \%$ de todas las muertes registradas, de estas muertes 7,4 millones se debieron a la cardiopatía coronaria y 6,7 millones, a los accidentes cerebrovasculares ${ }^{4}$.

Para la Organización Mundial de la Salud, las enfermedades que lideran las muertes son las cardiovasculares, dentro de las cuales se destacan: la hipertensión arterial (13\% de las muertes a nivel mundial), el tabaquismo (9\%), la diabetes mellitus $(6 \%)$, el sedentarismo $(6 \%)$, el sobrepeso y la obesidad $(5 \%)^{5}$.
Las principales causas de mortalidad en Colombia por grandes grupos registradas en el período 1997-2010 están representadas en primer lugar por las enfermedades del sistema circulatorio, que osciló entre un 28 y un $30 \%$ del total de muertes ${ }^{6}$. Y según la Organización Mundial de la Salud, en el año 2011, la tasa de mortalidad de Colombia por cada 100 mil habitantes para las enfermedades cardiovasculares y la diabetes es de 166,7 para mujeres y de 205,9 para hombres ${ }^{7}$.

Las tasas de mortalidad por 100 mil habitantes de infarto agudo de miocardio entre 2005 y 2010 más elevadas se registraron en los departamentos de Tolima $(84,53)$, Caldas $(81,72)$, Quindío $(74,53)$, Risaralda $(70,50)$ y Huila $(68,17)^{7}$.

En el departamento del Quindío las muertes por enfermedades cardiovasculares son las causas con mayor frecuencia observadas en el período entre los años 2005 a 2011, cuyo máximo número de eventos se dieron en el año 2010 con 1.008 defunciones $^{8}$.

El riesgo cardiovascular es la probabilidad que tiene un individuo de sufrir una de estas enfermedades dentro de un determinado plazo de tiempo y esto va a depender fundamentalmente de la cantidad de factores de riesgo que estén presentes en el individuo.

En el año 1990 se propuso la primera escala de evaluación del riesgo cardiovascular la cual predice el riesgo individual de presentar un evento coronario en 10 años, derivado de la enfermedad ateroesclerótica. En la actualidad existen diferentes escalas de medición de riesgo cardiovascular como la Framingham y la Score Procam. 
La escala de evaluación de riesgo de Framingham por categorías estima el riesgo cardiovascular global, utilizando un método de puntuación con base a las siguientes variables: la edad, el sexo, el colesterol HDL, el colesterol total, la presión arterial sistólica, el tabaquismo y la diabetes; con la cual se puede calcular el riesgo coronario a los 10 años que incluye: la angina estable, el infarto agudo de miocardio y la muerte coronaria?.

La escala Procam está basada en una cohorte de 5.389 hombres de 35-65 años de edad, de Münster, Alemania, seguidos durante 10 años, en los cuales 325 presentaron eventos coronarios mayores (muerte coronaria e infarto no fatal). Utiliza las siguientes variables: edad, c-LDL, cHDL, triglicéridos, antecedente familiar de infarto, diabetes mellitus, tabaquismo y presión arterial sistólica. Mide el riesgo de infarto y muerte coronaria a los 10 años, esta tabla tiene una serie de peculiaridades que la diferencian del resto de métodos de cálculo de riesgo coronario ya que considera como variable el antecedente familiar de infarto agudo al miocardio y utiliza como variables independientes 3 factores lipídicos distintos del colesterol total ${ }^{9}$.

En el volumen 21 No. 4 de la Revista Colombiana de Cardiología del año 2014, se publica el estudio "Validación de los modelos de predicción de Framingham y Procam como estimadores del riesgo cardiovascular en una población colombiana", el cual brinda un factor de multiplicación para ajustar la escala a la población colombiana, en el caso de Framingham de 0,75 y en el caso de Procam 0,93, al valor final del riesgo total establecido ${ }^{10}$.

El presente estudio aplicó las escalas de riesgo cardiovascular en una población de un programa de riesgo cardiovascular en una institución de salud de la ciudad de Armenia, Quindío.

\section{Materiales y métodos}

Tipo de estudio: Se planteó un estudio de tipo descriptivo de corte transversal.

Población y muestra: Aproximadamente 100.000 pacientes de una IPS de primer nivel de atención en la ciudad de Armenia, Quindío. El 10\% de los pacientes que pertenecen al programa de riesgo cardiovascular, de los cuales se tomó una muestra estadísticamente significativa con un nivel de confianza del $95 \%$, margen de error del $5 \%$ y una heterogeneidad del $50 \%$, para un valor de $n=374$ pacientes.

Muestreo: Se realizó de forma aleatoria por medio del programa Microsoft Office Excel 2013, en caso de no cumplir con los criterios de inclusión o cumplir con los criterios de exclusión se eligió al inmediatamente siguiente en la lista de la base de datos.

La información de la base de datos del programa de riesgo cardiovascular se procesó en una hoja de Excel.

Criterios de inclusión: Se incluyeron en el estudio aquellos pacientes que en la base de datos contaron con las variables requeridas por las escalas de estimación del riesgo cardiovascular, las cuales se plantean a continuación: edad, sexo, colesterol LDL, colesterol HDL, colesterol total, triglicéridos, talla, peso, tensión arterial sistólica, diabetes mellitus, tabaquismo, antecedentes familiares de enfermedad cardiovascular.
Criterios de exclusión: Se excluyeron los registros de los pacientes que presentaron un evento cardiovascular previamente (infarto agudo de miocardio fatal, infarto agudo de miocardio no fatal, accidente cerebrovascular).

Se reemplazaron los registros que no llenaran los criterios enunciados y se eligió al inmediatamente siguiente en la lista de la base de datos.

Análisis de la información: El análisis de las variables obtenidas de la base de datos del centro de atención de la ciudad de Armenia, permitió conocer el riesgo cardiovascular con las escalas Framingham por categorías, Framingham Colombia, Procam y Procam Colombia de los pacientes pertenecientes al programa de riesgo cardiovascular de dicha entidad.

Para esto se utilizaron las escalas Framingham y Procam. Cálculo que se realizó de manera manual en el programa Microsoft Excel 2013. El ajuste dado en Colombia se realizó mediante los parámetros establecidos en el estudio de "Validación de los modelos de predicción de Framingham y Procam como estimadores del riesgo cardiovascular en una población colombiana"' los cuales arrojan un factor de multiplicación para ajustar la escala a población colombiana, en el caso de Framingham de 0,75 y en el caso de Procam 0,93 al valor final del riesgo total establecido ${ }^{10}$.

Después de obtenidos y tabulados los datos en la herramienta Excel, los datos se procesaron en la herramienta Statgraphics ${ }^{\circledR}$; en la cual se hizo la descripción de las variables por medidas de tendencia central. Se llevó a cabo una descripción de cada una de las variables, con promedio, desviación estándar e intervalos de confianza del 95\%. Y se estableció una relación de las variables del estudio con el riesgo cardiovascular determinado, mediante una regresión múltiple.

\section{Resultados}

\section{Población y muestra}

La población del estudio fueron los pacientes del programa de riesgo cardiovascular de un centro de atención en salud durante el año 2015.

De los 138.378 pacientes del centro de atención, 12.222 pertenecen al programa de riesgo cardiovascular en el año 2015 , lo que equivale a una prevalencia del $8,83 \%$.

Cada paciente que pertenece al programa de riesgo cardiovascular tuvo un promedio de 4,25 consultas en el año, lo que corresponde a una consulta trimestral.

Del total de pacientes del programa de riesgo cardiovascular 9.348 son hipertensos, lo cual corresponde al $76,48 \%$ y 2.749 son diabéticos que corresponden al $22,49 \%$ del programa de riesgo cardiovascular.

En la tabla 1, se puede observar que la edad promedio fue de 64,54 años. Dentro de las variables de las medidas antropométricas se encontró el peso en $67,33 \mathrm{~kg}$ promedio y la talla en 155,88 cm; del índice de masa corporal se calculó una media estadística de $27,71 \mathrm{~m}^{2} / \mathrm{kg}$, lo cual corresponde a un estado de sobrepeso.

Las variables correspondientes al perfil lipídico evidencian que la variable HDL presenta una media estadística correspondiente a $46,83 \mathrm{mg} / \mathrm{dL}$, la cual estaría en los rangos de normalidad, la variable LDL presenta una media 
Tabla 1 Variables identificadas en la población a estudio

\begin{tabular}{lll}
\hline Variable & $\begin{array}{l}\text { Media } \pm \\
\text { Desviación } \\
\text { Estándar }\end{array}$ & IC 95\% \\
\hline Peso kg & $67,33 \pm 14,14$ & $65,89-68,77$ \\
Talla cm & $155,88 \pm 9,36$ & $154,93-156,83$ \\
$\mathrm{IMC} \mathrm{kg/m²}$ & $27,71 \pm 5,42$ & $27,16-28,26$ \\
Edad Años & $64,54 \pm 12,80$ & $63,24-65,84$ \\
Colesterol HdL mg/dl & $46,83 \pm 12,39$ & $45,57-48,09$ \\
Colesterol LdL mg/dl & $126,16 \pm 44,11$ & $121,68-130,65$ \\
Triglicéridos mg/dl & $178,07 \pm 101,17$ & $167,78-188,36$ \\
Colesterol total mg/dl & $208,61 \pm 47,25$ & $203,80-213,41$ \\
Presión sistólica mm Hg & $125,47 \pm 17,25$ & $123,72-127,23$ \\
Presión diastólica mm Hg & $77,50 \pm 9,34$ & $76,55-78,45$ \\
\hline
\end{tabular}

estadística en el valor de $126,16 \mathrm{mg} / \mathrm{dL}$, la cual estaría en el rango limítrofe bajo, la variable que corresponde a los triglicéridos presenta su media estadística en el valor de $178,07 \mathrm{mg} / \mathrm{dL}$, la cual estaría en el rango de levemente elevado; la variable que corresponde al colesterol total presenta una media estadística de $208,6 \mathrm{mg} / \mathrm{dL}$ que se encuentra por encima de los rangos de normalidad en la clasificación limítrofe alto, todos estos valores de referencia basados en el ATP III ${ }^{11}$, (tabla 1 ).

La presión arterial se encontró en $125 / 77$ mm Hg en promedio, la presión arterial sistólica en una media estadística de $125,47 \mathrm{~mm} \mathrm{Hg}$ que corresponde a la clasificación normal alta, la media estadística para la variable presión arterial diastólica corresponde a $77,50 \mathrm{~mm} \mathrm{Hg}$ que se clasifica como óptima, estos valores tomados de la sociedad europea de hipertensión ESH/ESC ${ }^{12}$, (tabla 1).

Respecto a los factores de riesgo en los sujetos de estudio, en la tabla 2 se puede observar que el $72,19 \%$ de la muestra es de sexo femenino y $27,81 \%$ de sexo masculino. El $20,86 \%$ de los participantes tienen diagnóstico de diabetes mellitus y el $9,36 \%$ son fumadores.

Para la variable antecedente familiar de haber presentado un evento cardiovascular, se encontró que el 87,43\% no tienen antecedente familiar de eventos cardiovasculares fuertes. En la tabla 3, se observa el cálculo del riesgo cardiovascular según las escalas de Framingham, Framingham Colombia, Procam y Procam Colombia lo cual puso en evidencia que, el riesgo cardiovascular según la escala de Framingham presenta una media estadística cuyo valor es $11,36 \%$, este valor corresponde a riesgo moderado; al aplicar la escala de Framingham Colombia arrojó un valor cuya media estadística es del 8,52\%, el cual corresponde a riesgo bajo. Al determinar el riesgo cardiovascular según la escala de Procam en los 374 pacientes se presenta una media estadística del $9,44 \%$, este valor corresponde a un riesgo bajo y al aplicar la escala de Procam Colombia se encontró una media estadística del 8,81\% cuyo valor determina que el riesgo es bajo. Es decir, con la escala de Framingham el riesgo es moderado y con las demás el riesgo es bajo.

En la escala de Framingham, en riesgo bajo se encuentra en el $48,93 \%$, en riesgo moderado en el $35,02 \%$, en riesgo alto en el $11,76 \%$ y en riesgo muy alto en el $4,27 \%$. En la escala de Framingham Colombia en riesgo bajo se encuentra en el $75,13 \%$, en riesgo moderado en el $17,64 \%$, en riesgo alto en el $5,88 \%$ y en riesgo muy alto en el $1,33 \%$.

En la escala Procam en riesgo bajo se encuentra en el $63,10 \%$, en riesgo moderado en el $24,59 \%$, en riesgo alto en

Tabla 2 Factores de riesgo encontrados en los sujetos de estudio

\begin{tabular}{llrl}
\hline Variable & & Frecuencia & Porcentaje \\
\hline Sexo & Masculino & 104 & $27,81 \%$ \\
Diabetes & Femenino & 270 & $72,19 \%$ \\
Mellitus & Sí & 78 & $20,86 \%$ \\
Consumo de cigarrillo & No & 296 & $79,14 \%$ \\
Antecedente familiar de & Sí & 35 & $9,36 \%$ \\
eventos CV & No & 339 & $90,64 \%$ \\
& Sí & 47 & $12,57 \%$ \\
\hline
\end{tabular}

Tabla 3 Cálculo del riesgo cardiovascular según las escalas de Framingham, Framingham Colombia, Procam y Procam Colombia

\begin{tabular}{llrlrrr}
\hline $\begin{array}{l}\text { Cálculo del Riesgo } \\
\text { por Diferentes } \\
\text { Escalas }\end{array}$ & $\begin{array}{l}\text { Promedio } \\
\text { Desviación } \\
\text { Estándar }\end{array}$ & IC 95\% & $\begin{array}{l}\text { \% Riesgo } \\
\text { Bajo }\end{array}$ & $\begin{array}{l}\text { \% Riesgo } \\
\text { Moderado }\end{array}$ & $\begin{array}{l}\% \text { Riesgo } \\
\text { Alto }\end{array}$ & $\begin{array}{c}\text { \% Riesgo } \\
\text { Muy Alto }\end{array}$ \\
\hline $\begin{array}{l}\text { Framingham } \\
\text { Framingham }\end{array}$ & $11,36 \pm 8,25$ & 10,$52 ; 12,20$ & $48,93 \%$ & $35,02 \%$ & $11,76 \%$ & $4,27 \%$ \\
Colombia & $8,52 \pm 6,19$ & 7,$89 ; 9,15$ & $75,13 \%$ & $17,64 \%$ & $5,88 \%$ & $1,33 \%$ \\
Procam & & & & & $7,48 \%$ & $4,81 \%$ \\
Procam Colombia & $8,44 \pm 7,99$ & 8,$62 ; 10,25$ & $63,10 \%$ & $24,59 \%$ & $12,29 \%$ & $0 \%$ \\
\hline
\end{tabular}


Tabla 4 Comparación de variables según sexo

\begin{tabular}{|c|c|c|c|c|c|}
\hline Variable & $\begin{array}{l}\text { Masculino } \\
\text { Media } \pm \mathrm{DE}\end{array}$ & IC 95\% & $\begin{array}{l}\text { Femenino } \\
\text { Media } \pm \mathrm{DE}\end{array}$ & IC 95\% & $\begin{array}{l}\text { p valor (Chi } \\
\text { cuadrado) }\end{array}$ \\
\hline Peso kg & $71,71 \pm 14,35$ & $69,81-73,6$ & $65,64 \pm 13,72$ & $64,46-66,82$ & 0,0002 \\
\hline Talla cm & $163,98 \pm 7,15$ & $162,9-165,05$ & $152,76 \pm 8,17$ & $152,09-153,43$ & 0,0000 \\
\hline$I M C \mathrm{~kg} / \mathrm{m}^{2}$ & $26,56 \pm 4,37$ & $25,83-27,30$ & $28,15 \pm 5,72$ & $27,69-28,60$ & 0,0112 \\
\hline Edad años & $65,70 \pm 13,29$ & $63,95-67,44$ & $64,09 \pm 12,60$ & $63,01-65,17$ & 0,2777 \\
\hline Colesterol HDL mg/dl & $41,13 \pm 10,94$ & $39,50-42,75$ & $49,02 \pm 12,23$ & $48,01-50,03$ & 0,0000 \\
\hline Colesterol LDL mg/dl & $116,64 \pm 37,54$ & $110,68-122,61$ & $129,83 \pm 45,93$ & $126,13-133,53$ & 0,0094 \\
\hline Triglicéridos mg/dl & $194,65 \pm 95,93$ & $180,91-208,39$ & $171,69 \pm 102,57$ & $163,16-180,21$ & 0,0491 \\
\hline Colesterol total $\mathrm{mg} / \mathrm{dl}$ & $196,71 \pm 39,83$ & $190,33-203,08$ & $213,19 \pm 49,11$ & $209,24-217,15$ & 0,0024 \\
\hline Sistólica mm Hg & $127,38 \pm 23,70$ & $125,03-129,73$ & $124,74 \pm 13,99$ & $123,28-126,20$ & 0,1861 \\
\hline Diastólica mm Hg & $77,45 \pm 9,52$ & $76,18-78,73$ & $77,52 \pm 9,29$ & $76,73-78,31$ & 0,9503 \\
\hline
\end{tabular}

Tabla 5 Cálculo del riesgo cardiovascular según las escalas de Framingham, Framingham Colombia, Procam y Procam Colombia por sexo

\begin{tabular}{|c|c|c|c|c|c|c|c|}
\hline $\begin{array}{l}\text { Cálculo del Riesgo } \\
\text { por Diferentes } \\
\text { Escalas }\end{array}$ & $\begin{array}{l}\text { Masculino } \\
\text { Media } \pm \text { DE }\end{array}$ & IC 95\% & $\begin{array}{l}\text { Clasificación } \\
\text { del Riesgo }\end{array}$ & $\begin{array}{l}\text { Femenino } \\
\text { Media } \pm \mathrm{DE}\end{array}$ & IC 95\% & $\begin{array}{l}\text { Clasificación } \\
\text { del Riesgo }\end{array}$ & $\begin{array}{l}\text { p Valor (Chi } \\
\text { Cuadrado) }\end{array}$ \\
\hline Framingham & $14,96 \pm 10,29$ & $13,87-16,04$ & Moderado & $9,97 \pm 6,86$ & $9,30-10,65$ & Bajo & 0,0000 \\
\hline $\begin{array}{l}\text { Framingham } \\
\text { Colombia }\end{array}$ & $11,22 \pm 7,72$ & $10,40-12,03$ & Moderado & $7,48 \pm 5,14$ & $6,97-7,98$ & Bajo & 0,0000 \\
\hline Procam & $10,47 \pm 8,49$ & $9,38-11,56$ & Moderado & $9,04 \pm 7,77$ & $8,36-9,71$ & Bajo & 0,1216 \\
\hline Procam Colombia & $9,78 \pm 7,93$ & $8,76-10,79$ & Bajo & $8,44 \pm 7,26$ & $7,81-9,07$ & Bajo & 0,1216 \\
\hline
\end{tabular}

el $7,48 \%$ y en riesgo muy alto en el $4,81 \%$. Y en la escala Procam Colombia en riesgo bajo se encuentra en el $72,19 \%$, en riesgo moderado en el $15,50 \%$, en riesgo alto en el $12,29 \%$ y en riesgo muy alto en el $0 \%$.

En la tabla 4, se observa que la las variables antropométricas y del perfil lipídico presentaron diferencia estadísticamente significativa por sexo. El índice de masa corporal se encuentra en sobrepeso para los hombres y las mujeres, siendo mayor en las mujeres $(p=0,0112)$ : la variable HDL se encuentra por debajo de lo normal para los hombres y en la normalidad para las mujeres $(p=0,0000)$; la variable LDL se encuentra en limítrofe bajo en las mujeres y limítrofe alto en los hombres $(p=0,0094)$, según ATP III.

Las variables de la presión arterial fueron normal alta para los hombres y las mujeres sin diferencias por sexo.

En la tabla 5, se observa el cálculo del riesgo cardiovascular según las escalas de Framingham, Framingham Colombia, Procam y Procam Colombia por sexo. Se observó una diferencia estadísticamente significativa en la clasificación del riesgo para los hombres y las mujeres, según Framingham y Framingham Colombia el riesgo fue moderado para los hombres y bajo para las mujeres $(p=0,0000)$.

En la escala Procam, el riesgo fue moderado para los hombres y bajo para las mujeres y en la escala Procam Colombia el riesgo fue bajo para los hombres y las mujeres, la diferencia no fue significativa por sexo.

En la tabla 6, al identificar los factores que determinan el riesgo cardiovascular en las diferentes escalas, mediante una regresión múltiple, el índice de masa corporal, la edad y el colesterol LDL, son variables estadísticamente significativas para la valoración del riesgo en las escalas Procam y Procam Colombia y no lo son para Framingham y Framingham Colombia.

El sexo, el colesterol HDL y la presión diastólica no son estadísticamente significativos para la valoración del riesgo en la escalas de Procam y Procam Colombia.

Los antecedentes familiares de eventos cardiovasculares no fueron estadísticamente significativos en ninguna escala.

\section{Discusión}

En la determinación del riesgo cardiovascular, en la población a estudio perteneciente a un programa de riesgo cardiovascular en una institución de salud en la población de Armenia, se encontró que, el riesgo cardiovascular fue de $11,36 \%$ según la escala de Framingham, lo cual equivale a riesgo moderado. Según Machado y Cols., el riesgo cardiovascular en los pacientes con dislipidemia afiliados al sistema de salud en Colombia fue de $14 \%$ en el año $2010^{13}$, que es mayor que el calculado obtenido en el presente estudio. No obstante, cuando se clasifica el riesgo, ambos equivalen a riesgo cardiovascular moderado (10-20\%). Y según el estudio de Navarro y Cols., el riesgo cardiovascular en adultos con síndrome metabólico en la ciudad de Soledad, Atlántico, por Framingham fue del 3\%, que equivale a riesgo bajo ${ }^{14}$.

En el presente estudio el riesgo cardiovascular es mayor en la población masculina con $14,96 \%$ (riesgo moderado), 
Tabla 6 Factores que determinan el riesgo cardiovascular en las diferentes escalas

\begin{tabular}{|c|c|c|c|c|}
\hline Variables & Framingham & $\begin{array}{l}\text { Framingham } \\
\text { Colombia }\end{array}$ & Procam & Procam Colombia \\
\hline IMC & $\begin{array}{l}\mathrm{p}=0,4851 \\
\text { Razón } \mathrm{F}: 1,01\end{array}$ & $\begin{array}{l}\mathrm{p}=0,4851 \\
\text { Razón } \mathrm{F}: 1,01\end{array}$ & $\begin{array}{l}\mathrm{p}=0,0001 \\
\text { Razón F: } 2,07\end{array}$ & $\begin{array}{l}\mathrm{p}=0,0001 \\
\text { Razón F: } 2,07\end{array}$ \\
\hline Edad & $\begin{array}{l}p=0,0806 \\
\text { Razón F: } 1,31\end{array}$ & $\begin{array}{l}\mathrm{p}=0,0806 \\
\text { Razón F: } 1,31\end{array}$ & $\begin{array}{l}\mathrm{p}=0,0000 \\
\text { Razón F: } 2,63\end{array}$ & $\begin{array}{l}\mathrm{p}=0,0000 \\
\text { Razón F: } 2,63\end{array}$ \\
\hline Sexo & $\begin{array}{l}\mathrm{p}=0,0000 \\
\text { Razón F: } 29,42\end{array}$ & $\begin{array}{l}\mathrm{p}=0,0000 \\
\text { Razón F: } 29,42\end{array}$ & $\begin{array}{l}\mathrm{p}=0,1216 \\
\text { Razón } \mathrm{F}: 2,41\end{array}$ & $\begin{array}{l}\mathrm{p}=0,1216 \\
\text { Razón F: 2,41 }\end{array}$ \\
\hline Colesterol HDL & $\begin{array}{l}\mathrm{p}=0,0078 \\
\text { Razón F: } 1,43\end{array}$ & $\begin{array}{l}\mathrm{p}=0,0078 \\
\text { Razón } \mathrm{F}: 1,43\end{array}$ & $\begin{array}{l}\mathrm{p}=0,6515 \\
\text { Razón } \mathrm{F}: 0,94\end{array}$ & $\begin{array}{l}\mathrm{p}=0,6515 \\
\text { Razón F: } 0,94\end{array}$ \\
\hline Colesterol LDL & $\begin{array}{l}\mathrm{p}=0,7889 \\
\text { Razón F: } 0,85\end{array}$ & $\begin{array}{l}\mathrm{p}=0,7889 \\
\text { Razón F: } 0,85\end{array}$ & $\begin{array}{l}\mathrm{p}=0,0025 \\
\text { Razón } \mathrm{F}: 1,95\end{array}$ & $\begin{array}{l}\mathrm{p}=0,0025 \\
\text { Razón F: } 1,95\end{array}$ \\
\hline Colesterol total & $\begin{array}{l}\mathrm{p}=0,0295 \\
\text { Razón } \mathrm{F}: 1,33\end{array}$ & $\begin{array}{l}\mathrm{p}=0,0295 \\
\text { Razón } \mathrm{F}: 1,33\end{array}$ & $\begin{array}{l}\mathrm{p}=0,0000 \\
\text { Razón } \mathrm{F}: 1,82\end{array}$ & $\begin{array}{l}\mathrm{p}=0,0000 \\
\text { Razón F: } 1,82\end{array}$ \\
\hline Triglicéridos & $\begin{array}{l}p=0,0074 \\
\text { Razón F: } 1,47\end{array}$ & $\begin{array}{l}\mathrm{p}=0,0074 \\
\text { Razón F: } 1,47\end{array}$ & $\begin{array}{l}\mathrm{p}=0,0223 \\
\text { Razón } \mathrm{F}: 1,37\end{array}$ & $\begin{array}{l}\mathrm{p}=0,0223 \\
\text { Razón } \mathrm{F}: 1,37\end{array}$ \\
\hline Sistólica & $\begin{array}{l}\mathrm{p}=0,0000 \\
\text { Razón F: } 2,69\end{array}$ & $\begin{array}{l}\mathrm{p}=0,0000 \\
\text { Razón F: } 2,69\end{array}$ & $\begin{array}{l}\mathrm{p}=0,0001 \\
\text { Razón } \mathrm{F}: 2,43\end{array}$ & $\begin{array}{l}\mathrm{p}=0,0001 \\
\text { Razón F: } 2,43\end{array}$ \\
\hline Diastólica & $\begin{array}{l}p=0,0044 \\
\text { Razón F: } 2,01\end{array}$ & $\begin{array}{l}\mathrm{p}=0,0044 \\
\text { Razón F: } 2,01\end{array}$ & $\begin{array}{l}\mathrm{p}=0,2194 \\
\text { Razón F: } 1,23\end{array}$ & $\begin{array}{l}\mathrm{p}=0,2194 \\
\text { Razón F: } 1,23\end{array}$ \\
\hline Diabetes Mellitus & $\begin{array}{l}\mathrm{p}=0,0000 \\
\text { Razón F: } 30,81\end{array}$ & $\begin{array}{l}\mathrm{p}=0,0000 \\
\text { Razón F: } 30,81\end{array}$ & $\begin{array}{l}\mathrm{p}=0,0000 \\
\text { Razón F: } 22,89\end{array}$ & $\begin{array}{l}\mathrm{p}=0,0000 \\
\text { Razón F: } 22,89\end{array}$ \\
\hline Consumo de cigarrillo & $\begin{array}{l}P=0,0147 \\
\text { Razón F: } 6,01\end{array}$ & $\begin{array}{l}\mathrm{p}=0,0147 \\
\text { Razón F: } 6,01\end{array}$ & $\begin{array}{l}\mathrm{p}=0,0000 \\
\text { Razón F: } 21,35\end{array}$ & $\begin{array}{l}\mathrm{p}=0,0000 \\
\text { Razón F: } 21,35\end{array}$ \\
\hline Antecedente familiar & $\begin{array}{l}\mathrm{p}=0,0255 \\
\text { Razón F: } 5,03\end{array}$ & $\begin{array}{l}\mathrm{p}=0,0548 \\
\text { Razón F: } 2,93\end{array}$ & $\begin{array}{l}\mathrm{p}=0,5801 \\
\text { Razón F: } 0,31\end{array}$ & $\begin{array}{l}\mathrm{p}=0,5801 \\
\text { Razón F: } 0,31\end{array}$ \\
\hline
\end{tabular}

frente a 9,97\% en las mujeres (riesgo bajo); a pesar que la población sujeto corresponde a mujeres en un $72,19 \%$, con un promedio de edad de 64,09 años, es decir postmenopáusicas. Navarro encontró que el riesgo cardiovascular es mayor en los hombres $(5,67 \%)$ que en las mujeres $(1,01 \%)$, riesgo bajo en ambos $\operatorname{casos}^{14}$.

La prevalencia de hipertensión arterial en el presente estudio fue de $76,48 \%$ en una población de riesgo cardiovascular; para la misma población en el año 2014, la prevalencia fue de $84,36 \%$, según Bedoya y Cols. ${ }^{15}$, no obstante, para estos autores el estudio estaba referido a la prevalencia del infarto de miocardio, el cual se excluyó en el presente estudio. En tanto que en el estudio Caribe, realizado en la población general en el año 2005, el $21 \%$ tenía diagnóstico de hipertensión arterial ${ }^{16}$. En la población general, el plan decenal en salud identificó una prevalencia de la hipertensión arterial de $22,8 \%$ para el año $2007^{9}$.

La prevalencia de la diabetes fue de $22,49 \%$ en este estudio, para la misma población de riesgo cardiovascular en el año 2014 la prevalencia fue de $21,84 \%{ }^{15}$. En Colombia se encontró que la prevalencia de la diabetes fue de 2,6\% para el año 2007, según el plan decenal de salud ${ }^{9}$. En cambio, el estudio Cardiovascular Risk Factor Multiple Evaluation in Latin America, mostró que la diabetes se halló entre el 7 y $9 \%$ para la población general en las ciudades de México, Bogotá y Santiago de Chile ${ }^{17}$. Y según el estudio Caribe 5,3\% de la población presentaba esta enfermedad ${ }^{16}$.

La prevalencia del tabaquismo en la muestra del estudio fue de 9,36\%. Para Bedoya y Cols., en la misma población del programa de riesgo cardiovascular el 12,97\% eran fumadores el año anterior ${ }^{15}$. Para Alvarado y Cols., fue de $9,3 \%{ }^{18}$, en el hospital departamental Felipe Suárez de Salamina, Caldas, en el año 2012. Y en el estudio Caribe el 15\% eran consumidores del tabaco ${ }^{16}$, en la población general. A nivel nacional se describe una prevalencia del tabaquismo del $12,8 \%$ en el plan decenal de salud para el año $2007^{9}$.

En la población a estudio el índice de masa corporal se calculó en una media de $27,71 \mathrm{~m}^{2} / \mathrm{kg}$, es decir, en sobrepeso; para Lombo y Cols., el índice de masa corporal fue de $27,75 \mathrm{~kg} / \mathrm{m}^{2}$, en una institución de salud de tercer nivel mostró, además, que la prevalencia del síndrome metabólico aumenta con el índice de masa corporal y se observó una prevalencia de síndrome metabólico de $25,5 \%$ en los pacientes con sobrepeso, según los criterios del ATP III, esta prevalencia aumentó a 78,17\% en el grupo de sobrepeso si se emplean los criterios de la American Heart Association ${ }^{19}$. Esto indica la importancia del índice de masa corporal como factor de riesgo cardiovascular.

Con la evaluación de los factores que determinan el riesgo cardiovascular en las diferentes escalas, el índice de masa corporal no fue determinante en las escalas Framingham y Framingham Colombia $(p=0,4851)$.

En las variables del perfil lipídico se halló que el colesterol LDL fue determinante para la valoración del riesgo cardiovascular con las escalas Procam y Procam Colombia $(p=0,0025)$ no así con las escala de Framingham y Framingham Colombia ( $p=0,7889$ ), la media estadística de este fue de $126,16 \mathrm{mg} / \mathrm{dL}$. El cual para la misma población en el año 2014 fue de $110,04 \mathrm{mg} / \mathrm{dL}^{15}$. 
Según Patiño-Villada y Cols., los factores de riesgo más importantes en una población urbana de Colombia fueron: la baja actividad física, la obesidad, la dislipidemia, el tabaquismo y el síndrome metabólico ${ }^{20}$. Estudios observacionales han mostrado una relación directamente proporcional entre el riesgo de enfermedad coronaria y las concentraciones séricas de colesterol, identificando a la hipercolesterolemia como un factor de riesgo independiente para los eventos cardiovasculares y los cerebrovasculares en personas con o sin historia de enfermedad cardiovascular. Por lo cual, la corrección del perfil lipídico, especialmente del colesterol de baja densidad (CLDL), constituye un objetivo terapéutico importante ${ }^{21}$.

La media de los triglicéridos fue de $178,07 \mathrm{mg} / \mathrm{dL}$, (levemente elevado). Para Lombo y Cols., los niveles de los triglicéridos promedio de la totalidad de pacientes fueron de $164,24 \mathrm{mg} / \mathrm{dL}^{19}$. Y para Bedoya y Cols., los triglicéridos en el mismo programa de riesgo cardiovascular de Armenia, Quindío, en el año 2014, fueron de $188,09 \mathrm{mg} / \mathrm{dL}^{15}$.

El colesterol HDL presenta una media de $46,83 \mathrm{mg} / \mathrm{dL}$ que equivale a la normalidad ${ }^{11}$, con un valor promedio de $41,13 \mathrm{mg} / \mathrm{dL}$ para los hombres y $49,02 \mathrm{mg} / \mathrm{dL}$ en las mujeres, en el estudio de Lombo y Cols., la media de los niveles de HDL fue de $51,25 \mathrm{mg} / \mathrm{dL}$, y se observaron más bajos en los hombres $(46,54 \mathrm{mg} / \mathrm{dL})$, que en las mujeres $(52,89 \mathrm{mg} / \mathrm{dL})^{19}$. Para la presión arterial sistólica se encontró una media de $125,47 \mathrm{~mm} \mathrm{Hg}$ (normal alta). Y la presión arterial diastólica en 77,50 mm Hg (óptima); en la misma población para el año 2014 la media de la presión arterial sistólica fue de $126,71 \mathrm{~mm} \mathrm{Hg}$ y diastólica de $76,60 \mathrm{~mm} \mathrm{Hg}{ }^{15}$. Para Lombo la presión arterial sistólica media fue de $126,35 \mathrm{~mm} \mathrm{Hg}$ y la presión diastólica fue de $80,12 \mathrm{~mm} \mathrm{Hg}^{19}$.

En este estudio se encontró una presión arterial sistólica promedio en los hombres de $127,38 \mathrm{~mm} \mathrm{Hg}$ y en las mujeres de $124,74 \mathrm{~mm} \mathrm{Hg}$. Para Bedoya y Cols., la presión arterial sistólica promedio en los hombres es de 124,06 mm $\mathrm{Hg}$ y en las mujeres es de $130,31 \mathrm{~mm} \mathrm{Hg}^{15}$. Es de anotar que en esta población de riesgo cardiovascular, la presión arterial promedio se encuentra en cifras normales, es decir, se encuentra controlada.

Al identificar los factores de riesgo para cada una de las escalas, en el presente estudio se evidencia que algunos de ellos son prevalentes y muy importantes en nuestra población, no son significativos en la determinación del riesgo cardiovascular en la población a estudio (tales como edad, índice de masa corporal y c-LDL), razón por la cual las escalas de Framingham y Framingham Colombia deben ser utilizadas con precaución en esta población. No obstante, es importante resaltar que el presente estudio tomó la población de un programa de riesgo cardiovascular en una institución de salud.

En cambio las escalas de Procam y Procam Colombia identifican más variables importantes como significativas. Si bien en la estimación de la escala de Procam la presión diastólica y el colesterol HDL no son significativos, es de considerar que para la escala de Procam la presión sistólica es más determinante como factor de riesgo y en el caso del colesterol $\mathrm{HDL}$, es de las variables que más tempranamente se alteran en toda la población a estudio, razón por la cual no hace la diferencia en este caso.

Al revisar el estudio de Múñoz y Cols., en el año $2014^{10}$, quien comparó las mismas escalas en una cohorte de población de prevención primaria en el hospital militar central, sugiere que la escala de Framingham debería usarse con precaución en la población colombiana de riesgo bajo e intermedio, sin historia previa de riesgo cardiovascular, dado que sobrestima el riesgo y tiene baja capacidad de discriminación. Y sugiere el uso de la función de riesgo de Procam ajustada por el sexo como una mejor opción para estimar el riesgo cardiovascular dura.

En la población a estudio, de manera similar que la propuesta de Múñoz, las escalas de Procam y Procam Colombia serían las más adecuadas para estimación del riesgo cardiovascular y no se desconocerían las variables de gran significancia y las otras escalas deben usarse con precaución en esta población.

\section{Conclusiones}

En una población de un programa de riesgo cardiovascular en una institución de salud de la ciudad de Armenia, Quindío, el presente estudio identificó variación en el riesgo cardiovascular, dependiendo de la escala de medición utilizada: en la escala de Framingham correspondió a riesgo moderado, con la escala de Framingham Colombia pasó a riesgo bajo. Con las escalas de Procam y Procam Colombia se encontró un riesgo bajo en ambas. En la clasificación del riesgo según Framingham y Framingham Colombia el riesgo fue moderado para los hombres y bajo para las mujeres; con las escalas Procam y Procam Colombia no hubo diferencia significativa por el sexo.

Al identificar los factores de riesgo para cada una de las escalas, se evidencia que el índice de masa corporal, la edad y el colesterol LDL, son variables estadísticamente significativas para la valoración del riesgo en las escalas Procam y Procam Colombia no así para Framingham y Framingham Colombia. El sexo, el colesterol HDL y la presión arterial diastólica no resultan estadísticamente significativos para la valoración del riesgo en la escala de Procam y Procam Colombia.

Por lo anterior, se propone que la escala adecuada para medición del riesgo cardiovascular en esta población son las escalas de Procam y Procam Colombia, porque identifican casi todas las variables de manera significativa, no así las escalas de Framingham y Framingham Colombia, que deben ser utilizadas con precaución en esta población.

\section{Responsabilidades éticas}

Protección de personas y animales. Los autores declaran que para esta investigación no se han realizado experimentos en seres humanos ni en animales.

Confidencialidad de los datos. Los autores declaran que en este artículo no aparecen datos de pacientes.

Derecho a la privacidad y consentimiento informado. Los autores declaran que en este Artículo no aparecen datos de pacientes. 


\section{Conflicto de intereses}

Los autores declaran no tener ningún conflicto de intereses.

\section{Reconocimientos y agradecimientos}

A Red Salud Armenia ESE y a la doctora Diana Marcela Curtidor por su orientación en los aspectos bioéticos.

\section{Bibliografía}

1. Organización Panamericana de la Salud; prevención de las enfermedades cardiovasculares, directrices para la evaluación y el manejo del riesgo cardiovascular. Washington, D.C. OPS., 2010.

2. Factores de riesgo cardiovascular - Instituto del Corazón de Texas (Texas Heart Institute). Texasheart.org. 2016.

3. Instituto Nacional de Salud. Enfermedad cardiovascular: principal causa de muerte en Colombia. Boletín No. 1 del Observatorio Nacional de Salud. 2013; Vol 1:1-6.

4. Organización Mundial de la Salud. Las 10 causas principales de defunción en el mundo. Ginebra: centro de prensa OMS; 2014.

5. Gómez J. Cardiovascular morbidity and mortality in the world. Rev. Colomb. Cardiol. 2012;9:298-9.

6. Ministerio Nacional de Salud de Colombia. Plan Decenal de Salud Pública, PDSP. 2012: La salud en Colombia la construyes tú. 2013.

7. Principales causas de mortalidad en Colombia. Bogotá, Colombia: Ministerio de Salud y Protección Social. 2010.

8. Gobernación del Quindío, Secretaria de Salud Departamental. Análisis de la situación de salud en el departamento. 2012.

9. Álvarez Cosmea A. Las tablas de riesgo cardiovascular: Una revisión crítica. Medifam. 2001;11:20-51.

10. Múñoz O, Rodríguez N, Ruiz Á, Rondón M. Validación de los modelos de predicción de Framingham y Procam como estimadores del riesgo cardiovascular en una población colombiana. Rev. Colomb. Cardiol. 2014;21:202-12.

11. Expert Panel on Detection, Evaluation, and Treatment of High Blood Cholesterol in Adults. Executive Summary of the Third Report of the National Cholesterol Education Program (NCEP) Expert Panel on Detection, Evaluation, and Treatment of High
Blood Cholesterol in Adults (Adult Treatment Panel III). JAMA. 2001;285:2486-97.

12. Mancia G, Fagard R, Narkiewicz K, Redón J, Zanchetti A, Böhm $M$, et al. ESH/ESC Guidelines for the management of arterial hypertension: the Task Force for the management of arterial hypertension of the European Society of Hypertension (ESH) and of the European Society of Cardiology (ESC). J. Hypertens. 2013;31:1281-357.

13. Machado-Alba Jorge E, Machado-Duque Manuel E. Prevalencia de factores de riesgo cardiovascular en pacientes con dislipidemia afiliados al sistema de salud en Colombia. Rev. Perú Med. Exp. Salud Pública. 2013;30:205-11.

14. Navarro E, Vargas R. Riesgo coronario según ecuación de Framingham en adultos con síndrome metabólico de la ciudad de Soledad, Atlántico. 2010. Rev. Colomb. Cardiol. 2012;19:109-18.

15. Bedoya-Ríos C, Mendoza-Lozano J, Nieto-Cárdenas OA. Prevalencia de infarto de miocardio en un programa de riesgo cardiovascular de una institución prestadora de salud en Armenia. Quindío. Rev. Colomb. Cardiol. 2016.

16. Fernando M, Arrieta C. Sociological study for detection of risk factors of cardiovascular diseases in the Colombian Caribbean Coast population. Rev. Colomb. Cardiol. 2005;12:122-8.

17. Schargrodsky H, Hernández R, Champagne BM, Silva H, Vinueza R, Silva Ayçaguer LC, et al., CARMELA Study Investigators. CARMELA: assessment of cardiovascular risk in seven Latin American cities. Am J. Med. 2008;121:58-65.

18. Alvarado C, Molina D, Zárate A, Toro E. Estudio EPRAS: estudio poblacional del riesgo cardiovascular de una población colombiana. Rev. Colomb. Cardiol. 2014;21:284-93.

19. Lombo B, Villalobos C, Tique C, Satizábal C, Franco AC. Metabolic syndrome prevalence in patients attending the hypertension clinic at the Fundación Santa Fe de Bogotá. Rev. Colomb. Cardiol. 2006;12:472-8.

20. Patiño-Villada F, Arango-Vélez E, Quintero-Velásquez M, ArenasSosa M. Cardiovascular risk factors in an urban Colombia population. Revista de Salud Pública. 2011;13:433-45.

21. Múñoz O, García Á, Fernández D, Higuera A, Ruiz Á, Aschner $P$, et al. Guía de práctica clínica para la prevención, detección temprana, diagnóstico, tratamiento y seguimiento de las dislipidemias: tratamiento farmacológico con estatinas. Rev. Colomb. Cardiol. 2015;22:14-21. 